\title{
Nonlinear Shear-free Radiative Collapse
}

\author{
S.S. Misthry ${ }^{* \dagger}$, S.D. Maharaj* and P.G.L. Leach, \\ Astrophysics and Cosmology Research Unit, \\ School of Mathematical Sciences, University of KwaZulu-Natal, \\ Private Bag X54001, Durban 4000, South Africa
}

November 5, 2018

\begin{abstract}
We study realistic models of relativistic radiating stars undergoing gravitational collapse which have vanishing Weyl tensor components. Previous investigations are generalised by retaining the inherent nonlinearity at the boundary. We transform the boundary condition to an Abel equation of the first kind. A variety of nonlinear solutions are generated all of which can be written explicitly. Several classes of infinite solutions exist.
\end{abstract}

Keywords: astrophysics; Einstein equations; radiating stars.

AMS numbers: 83 C 15; 83 C 22; 85 A 99

\section{Introduction}

The evolution of a radiating star undergoing gravitational collapse, in the context of general relativity, has occupied the attention of researchers in astrophysics in recent times. The derivation of the junction conditions by Santos [1] has made it possible to obtain exact models of an interior spacetime with heat flux to match with the exterior Vaidya spacetime; at the boundary of the star the radial pressure is nonzero. A variety of exact solutions has been generated over the years to study the cosmic censorship hypothesis, gravitational collapse with dissipation, end state of superdense matter, dynamical stability of radiating matter and temperature profiles in

${ }^{*}$ SSM and SDM dedicate this work to Peter Leach on his $65^{\text {th }}$ birthday

${ }^{\dagger}$ Permanent address: Department of Mathematics, Durban University of Technology, Steve Biko Campus, Durban, 4001 
the context of irreversible thermodynamics. De Oliviera et al. [2] proposed a radiating model in which an initial static configuration leads to collapse. This approach may be adapted to study the end state of collapse as shown by Govender et al. [3]. Kolassis et al. [4] assumed that the fluid trajectories are geodesic and generated exact solutions. These assumptions lead to particular solutions which may be used to study the physical features of the model such as the relaxational effects of the collapsing fluid on the temperature profile in theories of causal thermodynamics [5]-[10].

In a recent treatment Herrera et al. [11] proposed a model in which the form of the Weyl tensor was highlighted when studying radiative collapse. This approach has the advantage of simplifying the Einstein field equations. However, Herrera et al. were not able to solve the junction conditions; only an approximate solution was found. Maharaj and Govender [12] showed that it is possible to solve the field equations and the junction conditions exactly. Their solution is expressible in terms of elementary functions and contains the Friedmann dust solution as a special case. It is interesting to note that Herrera et al. [13] showed that other classes of solutions in terms of the elementary functions are possible. The exact solutions in both [12] and [13] depend upon the introduction of a transformation that linearises the boundary condition. The purpose of this paper is to demonstrate that it is possible to obtain other models by transforming the boundary condition to an Abel's equation which is necessarily nonlinear. We explicitly find exact solutions to the Abel equation under particular assumptions and thereby demonstrate that conformally flat radiating stars contain a richer structure than previously suspected.

The main objective of this paper is to show that we can generate radiating relativistic stellar models without having to eliminate the nonlinearity at the boundary. In Section 2, we describe the basic features of the model for a radiating star and present the relevant differential equations. Results generated in previous investigations are briefly discussed in Section 3. These have been obtained by introducing a transformation that leads to a linear equation at the boundary. In Section 4, we introduce a new transformation at the boundary that leads to an Abel's equation. We show explicitly that a variety of exact solutions can be generated from the Abel equation. Consequently a variety of new models for radiating relativistic stars, with vanishing Weyl stresses, are possible. The physical features of the solutions are briefly considered in Section 5 .

\section{The Model}

We consider a spherically symmetric radiating star undergoing shear-free gravitational collapse. The line element for shear-free matter interior to the boundary of the radiating star is given by

$$
d s^{2}=-A^{2} d t^{2}+B^{2}\left[d r^{2}+r^{2}\left(d \theta^{2}+\sin ^{2} \theta d \phi^{2}\right)\right]
$$


where $A=A(t, r)$ and $B=B(t, r)$ are the metric functions. The energy momentum tensor including radiation for the interior spacetime is

$$
T_{a b}=(\rho+p) u_{a} u_{b}+p g_{a b}+q_{a} u_{b}+q_{b} u_{a}
$$

where the energy density $\rho$, the pressure $p$ and the heat flow vector $q_{a}$ are measured relative to the timelike fluid 4 -velocity $u^{a}=\frac{1}{A} \delta_{0}^{a}$. The heat flow vector assumes the form $q^{a}=(0, q, 0,0)$ since $q^{a} u_{a}=0$ for radially directed heat flow.

The nonzero components of the Einstein field equations, for the line element (11) and the energy momentum (21), can be written as

$$
\begin{aligned}
\rho= & \frac{3}{A^{2}} \frac{\dot{B}^{2}}{B^{2}}-\frac{1}{B^{2}}\left(2 \frac{B^{\prime \prime}}{B}-\frac{B^{\prime 2}}{B^{2}}+\frac{4}{r} \frac{B^{\prime}}{B}\right) \\
p= & \frac{1}{A^{2}}\left(-2 \frac{\ddot{B}}{B}-\frac{\dot{B}^{2}}{B^{2}}+2 \frac{\dot{A}}{A} \frac{\dot{B}}{B}\right) \\
& +\frac{1}{B^{2}}\left(\frac{B^{\prime 2}}{B^{2}}+2 \frac{A^{\prime}}{A} \frac{B^{\prime}}{B}+\frac{2}{r} \frac{A^{\prime}}{A}+\frac{2}{r} \frac{B^{\prime}}{B}\right) \\
p= & -2 \frac{1}{A^{2}} \frac{\ddot{B}}{B}+2 \frac{\dot{A}}{A^{3}} \frac{\dot{B}}{B}-\frac{1}{A^{2}} \frac{\dot{B}^{2}}{B^{2}}+\frac{1}{r} \frac{A^{\prime}}{A} \frac{1}{B^{2}} \\
& +\frac{1}{r} \frac{B^{\prime}}{B^{3}}+\frac{A^{\prime \prime}}{A} \frac{1}{B^{2}}-\frac{B^{\prime 2}}{B^{4}}+\frac{B^{\prime \prime}}{B^{3}} \\
q= & -\frac{2}{A B^{2}}\left(-\frac{\dot{B}^{\prime}}{B}+\frac{B^{\prime} \dot{B}}{B^{2}}+\frac{A^{\prime}}{A} \frac{\dot{B}}{B}\right)
\end{aligned}
$$

The Weyl tensor has all components proportional to

$$
C_{2323}=\frac{r^{4}}{3} B^{2} \sin ^{2} \theta\left[\left(\frac{A^{\prime}}{A}-\frac{B^{\prime}}{B}\right)\left(\frac{1}{r}+2 \frac{B^{\prime}}{B}\right)-\left(\frac{A^{\prime \prime}}{A}-\frac{B^{\prime \prime}}{B}\right)\right]
$$

according to

$$
\begin{aligned}
C_{2323} & =-r^{4}\left(\frac{B}{A}\right)^{2} \sin ^{2} \theta C_{0101}=2 r^{2}\left(\frac{B}{A}\right)^{2} \sin ^{2} \theta C_{0202} \\
& =2 r^{2}\left(\frac{B}{A}\right)^{2} C_{0303}=-2 r^{2} \sin ^{2} \theta C_{1212}=-2 r^{2} C_{1313}
\end{aligned}
$$

which represent the tidal forces. For conformal flatness these components must all vanish so that $C_{2323}=0$. This leads to a nonlinear partial differential equation which is easily solved so that

$$
A=\left(C_{1}(t) r^{2}+1\right) B
$$


Now from (3b) and (3C), and using (44), we obtain

$$
\frac{B^{\prime \prime}}{B^{\prime}}-2 \frac{B^{\prime}}{B}-\frac{1}{r}=0
$$

which is the condition of pressure isotropy. Equation (5) is integrable and we get

$$
B=\frac{1}{C_{2}(t) r^{2}+C_{3}(t)}
$$

where $C_{1}(t), C_{2}(t)$ and $C_{3}(t)$ are functions of time. The forms for the metric functions $A$ and $B$ given above generate an exact solution to the Einstein field equations (3).

The interior spacetime (11) has to be matched across the boundary $r=b$ to the exterior Vaidya spacetime

$$
d s^{2}=-\left(1-\frac{2 m(v)}{R}\right) d v^{2}-2 d v d R+R^{2}\left(d \theta^{2}+\sin ^{2} \theta d \phi^{2}\right)
$$

The hypersurface at the boundary is denoted by $\Sigma$. The junction conditions at $\Sigma$ have the form

$$
\begin{aligned}
(A d t)_{\Sigma} & =\left[\left(1-\frac{m}{R}+2 \frac{d R}{d v}\right)^{1 / 2} d v\right]_{\Sigma} \\
(r B)_{\Sigma} & =R_{\Sigma} \\
p_{\Sigma} & =(q B)_{\Sigma} \\
{[m(v)]_{\Sigma} } & =\left[\frac{r^{3}}{2}\left(\frac{\dot{B}^{2} B}{A^{2}}-\frac{B^{\prime 2}}{B}\right)-r^{2} B^{\prime}\right]_{\Sigma}
\end{aligned}
$$

on matching (1) and (17). For our model the junction conditions (8) reduce to the following nonlinear ordinary differential equation

$$
\begin{array}{r}
\ddot{C}_{2} b^{2}+\ddot{C}_{3}-\frac{3}{2} \frac{\left(\dot{C}_{2} b^{2}+\dot{C}_{3}\right)^{2}}{C_{2} b^{2}+C_{3}}-\frac{\dot{C}_{1} b^{2}\left(\dot{C}_{2} b^{2}+\dot{C}_{3}\right)}{C_{1} b^{2}+1}-2\left(\dot{C}_{3} C_{1}-\dot{C}_{2}\right) b \\
+2 \frac{C_{1} b^{2}+1}{C_{2} b^{2}+C_{3}}\left[C_{2}\left(C_{2}-2 C_{1} C_{3}\right) b^{2}+C_{3}\left(C_{1} C_{3}-2 C_{2}\right)\right]=0
\end{array}
$$

resulting from the (nonvanishing) pressure gradient across the hypersurface $\Sigma$. Equation (9) governs the evolution of a radiating star with vanishing Weyl stresses. To complete the description of this radiating model we need to solve the remaining junction condition (9).

\section{Elementary solutions}

The governing equation (9) is a highly nonlinear equation presenting a formidable mathematical task to solve exactly in general. Note that in previous attempts to integrate (9) assumptions 
were made that effectively linearised this boundary condition. We briefly summarise the known results.

Herrera et al. [11] assumed the following approximate forms for the temporal functions

$$
C_{1}=\epsilon c_{1}(t), \quad C_{2}=0, \quad C_{3}=\frac{a}{t^{2}},
$$

where $0<\epsilon<<1$ and $a>0$, is a constant. With the assumptions contained in (10), (9) yields the approximate solution

$$
C_{1} \approx C_{1}(0) \exp \left(\frac{-t^{2}}{2 b^{2}}-\frac{2 t}{b}\right)
$$

Note that on setting $C_{1}=0$ the solution reduces to a collapsing Friedmann dust sphere.

Maharaj and Govender [12] were the first to determine a closed form solution for (9). They assumed that $C_{1}=C$ (a constant), $C_{2}=0$ and introduced the transformation $C_{3} \equiv u^{-2}$ so that (9) takes the linear form

$$
\ddot{u}-2 C b \dot{u}-\left(C b^{2}+1\right) C u=0
$$

in the new variable $u$. Three categories of closed form solutions in terms of the elementary functions were obtained depending on the nature of the roots of the characteristic equation.

Herrera et al. [13] extended this treatment to obtain a wider class of solutions. They set $C_{1}=C$ (a constant), $C_{2}=\alpha C_{3}$ and introduced the transformation $C_{3}(t)=u^{-2}(t)$ so that (9) can be written as

$$
\ddot{u}-\frac{2(C-\alpha) b}{\alpha b^{2}+1} \dot{u}-\frac{\left(C b^{2}+1\right)}{\left(\alpha b^{2}+1\right)^{2}}\left[\alpha(\alpha-2 C) b^{2}+(C-2 \alpha)\right] u=0
$$

which is linear in $u$. Then equation (11) admits three classes of solution given by

Case 1: $(C-\alpha)^{2} b^{2}+\left(C b^{2}+1\right)\left[\alpha(\alpha-2 C) b^{2}+(C-2 \alpha)\right]>0$

$$
\begin{aligned}
C_{3}(t) & =\left[\beta_{1} \exp \left(\frac{(C-\alpha) b+\sqrt{(C-\alpha)^{2} b^{2}+\left(C b^{2}+1\right)\left[\alpha(\alpha-2 C) b^{2}+(C-2 \alpha)\right]}}{\alpha b^{2}+1}\right) t\right. \\
& \left.+\beta_{2} \exp \left(\frac{(C-\alpha) b-\sqrt{(C-\alpha)^{2} b^{2}+\left(C b^{2}+1\right)\left[\alpha(\alpha-2 C) b^{2}+(C-2 \alpha)\right]}}{\alpha b^{2}+1}\right) t\right]^{-2}
\end{aligned}
$$

Case 2: $(C-\alpha)^{2} b^{2}+\left(C b^{2}+1\right)\left[\alpha(\alpha-2 C) b^{2}+(C-2 \alpha)\right]<0$

$$
\begin{array}{r}
C_{3}(t)=\left[\operatorname { e x p } ( \frac { ( C - \alpha ) b } { \alpha b ^ { 2 } + 1 } t ) \left(\beta_{1} \cos \left(\frac{\sqrt{(C-\alpha)^{2} b^{2}+\left(C b^{2}+1\right)\left[\alpha(\alpha-2 C) b^{2}+(C-2 \alpha)\right]}}{\left(\alpha b^{2}+1\right)}\right) t\right.\right. \\
\left.\left.+\beta_{2} \sin \left(\frac{\sqrt{(C-\alpha)^{2} b^{2}+\left(C b^{2}+1\right)\left[\alpha(\alpha-2 C) b^{2}+(C-2 \alpha)\right]}}{\left(\alpha b^{2}+1\right)}\right) t\right)\right]^{-2}
\end{array}
$$


Case 3: $(C-\alpha)^{2} b^{2}+\left(C b^{2}+1\right)\left[\alpha(\alpha-2 C) b^{2}+(C-2 \alpha)\right]=0$

$$
C_{3}(t)=\left(\beta_{1}+\beta_{2} t\right)^{-2} \exp \left(\frac{-2(C-\alpha) b}{\alpha b^{2}+1} t\right)
$$

where $\beta_{1}$ and $\beta_{2}$ are constants of integration. These solutions reduce to the Maharaj and Govender [12] model when $\alpha=0$. Note that other transformations that linearise (9) are possible as indicated in [13].

\section{Abel Equation}

The nonlinearity and complexity in the boundary condition (9) is clearly evident. It is therefore remarkable that closed form solutions in terms of elementary functions have been shown to exist as shown in Section 3. These particular closed form solutions have been generated from linearised forms of the governing equation (9). A natural extension would be a study of the existence of nonlinear solutions to the differential equation (9). Such classes of solutions, if they exist, are important in the study of nonlinear behaviour of the shear-free, conformally flat model. Consequently we seek classes of solutions which retain the inherent nonlinear structure of (9). These have not been found in the past due to the inherent difficulties of coping with nonlinearity.

Here we consider a particular nonlinear transformation which leads to exact solutions. It is convenient to replace the function $C_{1}(t)$ with

$$
U=C_{1} b^{2}+1
$$

Then the governing equation (9) may be written with some rearrangement as

$$
\begin{gathered}
\dot{U}\left(\dot{C}_{2} b^{2}+\dot{C}_{3}\right)+U\left[\frac{3}{2} \frac{\left(\dot{C}_{2} b^{2}+\dot{C}_{3}\right)^{2}}{C_{2} b^{2}+C_{3}}-\frac{2}{b}\left(\dot{C}_{2} b^{2}+\dot{C}_{3}\right)-\left(\ddot{C}_{2} b^{2}+\ddot{C}_{3}\right)\right] \\
+2 U^{2}\left[\frac{\dot{C}_{3}}{b}-\frac{1}{C_{2} b^{2}+C_{3}}\left(C_{2}^{2} b^{2}-\frac{C_{3}^{2}}{b^{2}}\right)\right]+2 U^{3} \frac{2 C_{2} b^{2}-C_{3}}{C_{2} b^{2}+C_{3}} \cdot \frac{C_{3}}{b^{2}}=0
\end{gathered}
$$

Equation (13) is complicated, but has the generic structure

$$
\mathcal{A} \dot{U}+\mathcal{B} U+\mathcal{C} U^{2}+\mathcal{D} U^{3}=0
$$


where we have set

$$
\begin{aligned}
\mathcal{A} & =\dot{C}_{2} b^{2}+\dot{C}_{3} \\
\mathcal{B} & =\frac{3}{2} \frac{\left(\dot{C}_{2} b^{2}+\dot{C}_{3}\right)^{2}}{C_{2} b^{2}+C_{3}}-\frac{2}{b}\left(\dot{C}_{2} b^{2}+\dot{C}_{3}\right)-\left(\ddot{C}_{2} b^{2}+\ddot{C}_{3}\right) \\
\mathcal{C} & =2\left(\frac{\dot{C}_{3}}{b}-\frac{1}{C_{2} b^{2}+C_{3}}\left(C_{2}^{2} b^{2}-\frac{C_{3}^{2}}{b^{2}}\right)\right) \\
\mathcal{D} & =2\left(\frac{2 C_{2} b^{2}-C_{3}}{C_{2} b^{2}+C_{3}} \cdot \frac{C_{3}}{b^{2}}\right)
\end{aligned}
$$

The transformed equation (14) is an Abel's equation of the first kind in the variable $U$. Abelian equations are difficult to solve in general. However, the advantage of utilising the transformation (12) is that (14) is a first order differential equation in $U$. In the following we present a comprehensive mathematical treatment of (14) and derive several classes of solutions.

\subsection{Case 1: $\mathcal{A}=0$}

The restriction $\mathcal{A}=0$ immediately gives

$$
C_{2} b^{2}+C_{3}=\alpha
$$

where $\alpha$ is a constant of integration. Then (13) becomes

$$
2 U^{2}\left[\frac{\dot{C}_{3}}{b}-\frac{1}{\alpha}\left(C_{2}^{2} b^{2}-\frac{C_{3}^{2}}{b^{2}}\right)\right]+2 U^{3} \frac{2 C_{2} b^{2}-C_{3}}{\alpha} \cdot \frac{C_{3}}{b^{2}}=0
$$

which is an algebraic equation in $U$.

Two cases arise: $U=0$ or $U \neq 0$ in (16). We easily find:

$$
\begin{array}{ll}
C_{1}= \begin{cases}-\frac{1}{b^{2}} & , U=0 \\
\frac{\alpha}{C_{3}\left(2 \alpha-3 C_{3}\right)}\left(\frac{\alpha}{b^{2}}-\frac{4 C_{3}}{b^{2}}+\frac{3 C_{3}^{2}}{\alpha b^{2}}-\frac{\dot{C}_{3}}{b}\right) & , U \neq 0\end{cases} \\
C_{2}=\frac{\alpha-C_{3}}{b^{2}} & \\
C_{3}=\text { arbitrary function of time } &
\end{array}
$$

This solution is particularly attractive since we have an infinite choice of $C_{3}$ and no integration is required. 


\subsection{Case 2: $\mathcal{D}=0$}

With $\mathcal{D}=0$ we have two possibilities: either $2 C_{2} b^{2}-C_{3}=0$ or $C_{3}=0$.

We firstly consider $2 C_{2} b^{2}-C_{3}=0$. Then (13) becomes

$$
\dot{U}+U\left[\frac{3}{2} \frac{\dot{C}_{3}}{C_{3}}-\frac{2}{b}-\frac{\ddot{C}_{3}}{\dot{C}_{3}}\right]=-U^{2}\left[\frac{4}{3 b}+\frac{2}{3} \frac{C_{3}}{b^{2} \dot{C}_{3}}\right]
$$

This is a Bernoulli equation with solution

$$
U=\frac{\dot{C}_{3} C_{3}^{-3 / 2} e^{2 t / b}}{K-\frac{8}{3 b} e^{2 t / b} C_{3}^{-1 / 2}+\frac{6}{b^{2}} \int C_{3}^{-1 / 2} e^{2 t / b} d t}
$$

where $K$ is a constant of integration. Hence for this first case we have the solution

$$
\begin{aligned}
C_{1} & =\frac{1}{b^{2}}\left(\frac{\dot{C}_{3} C_{3}^{-3 / 2} e^{2 t / b}}{K-\frac{8}{3 b} e^{2 t / b} C_{3}^{-1 / 2}+\frac{6}{b^{2}} \int C_{3}^{-1 / 2} e^{2 t / b} d t}-1\right) \\
C_{2} & =\frac{C_{3}}{2 b^{2}} \\
C_{3} & =\text { arbitrary function of time }
\end{aligned}
$$

This is an infinite class of solutions depending on $C_{3}$.

Now we consider $C_{3}=0$. The Abel equation (13) becomes

$$
\dot{U}+U\left(\frac{3}{2} \frac{\dot{C}_{2}}{C_{2}}-\frac{2}{b}-\frac{\ddot{C}_{2}}{\dot{C}_{2}}\right)=2 U^{2} \frac{C_{2}}{\dot{C}_{2} b^{2}}
$$

This is again a Bernoulli equation with solution

$$
U=\frac{\dot{C}_{2} C_{2}^{-3 / 2} e^{2 t / b}}{K^{\prime}-\frac{2}{b^{2}} \int e^{2 t / b} C_{2}^{-1 / 2} d t}
$$

where $K^{\prime}$ is a constant of integration. Therefore for the second case we have the solution

$$
\begin{aligned}
C_{1} & =\frac{1}{b^{2}}\left(\frac{\dot{C}_{2} C_{2}^{-3 / 2} e^{2 t / b}}{K^{\prime}-\frac{2}{b^{2}} \int e^{2 t / b} C_{2}^{-1 / 2} d t}-1\right) \\
C_{2} & =\text { arbitrary function of time } \\
C_{3} & =0
\end{aligned}
$$

Again we have generated an infinite class of solutions depending on $C_{2}$. 


\subsection{Case 3: $\mathcal{C}=0$}

Upon setting $\mathcal{C}=0$ we obtain the equation

$$
\frac{\dot{C}_{3}}{b}-\frac{1}{C_{2} b^{2}+C_{3}}\left(C_{2}^{2} b^{2}-\frac{C_{3}^{2}}{b^{2}}\right)=0
$$

This equation is quadratic in $C_{2}$ which implies

$$
C_{2}=\frac{\dot{C}_{3} b \pm \sqrt{\dot{C}_{3}{ }^{2} b^{2}-4 C_{3}\left(C_{3}+\dot{C}_{3} b\right)}}{2 b^{2}}
$$

Hence $C_{2}$ is a known quantity if the function $C_{3}$ is specified.

The Abelian equation (13) has the form

$$
\begin{aligned}
& \dot{U}\left(\dot{C}_{2} b^{2}+\dot{C}_{3}\right)+U\left[\frac{3}{2} \frac{\left(\dot{C}_{2} b^{2}+\dot{C}_{3}\right)^{2}}{C_{2} b^{2}+C_{3}}-\frac{2}{b}\left(\dot{C}_{2} b^{2}+\dot{C}_{3}\right)\right. \\
& \left.-\left(\ddot{C}_{2} b^{2}+\ddot{C}_{3}\right)\right]=-2 U^{3}\left[\frac{2 C_{2} b^{2}-C_{3}}{C_{2} b^{2}+C_{3}} \cdot \frac{C_{3}}{b^{2}}\right]
\end{aligned}
$$

The equation is complicated, but may be written concisely as

$$
\alpha \dot{U}+\beta U=-\gamma U^{3}
$$

where

$$
\begin{aligned}
\alpha & =\dot{C}_{2} b^{2}+\dot{C}_{3} \\
\beta & =\frac{3}{2} \frac{\left(\dot{C}_{2} b^{2}+\dot{C}_{3}\right)^{2}}{C_{2} b^{2}+C_{3}}-\frac{2}{b}\left(\dot{C}_{2} b^{2}+\dot{C}_{3}\right)-\left(\ddot{C}_{2} b^{2}+\ddot{C}_{3}\right) \\
\gamma & =2 \frac{2 C_{2} b^{2}-C_{3}}{C_{2} b^{2}+C_{3}} \cdot \frac{C_{3}}{b^{2}}
\end{aligned}
$$

The simpler equation (20) has the form of a Bernoulli equation with solution

$$
\begin{aligned}
U & =\frac{1}{e^{\int(\beta / \alpha) d t}\left(\int \frac{2 \gamma}{\alpha} e^{-\int(2 \beta / \alpha) d t} d t\right)^{1 / 2}} \\
& =\frac{e^{(2 t / b)}\left(\dot{C}_{2} b^{2}+\dot{C}_{3}\right)}{\left(C_{2} b^{2}+C_{3}\right)^{3 / 2}\left[K^{\prime \prime}+\frac{4}{b^{2}} \int \frac{e^{(4 t / b)} C_{3}\left(2 C_{2} b^{2}-C_{3}\right)\left(\dot{C}_{2} b^{2}+\dot{C}_{3}\right)}{\left(C_{2} b^{2}+C_{3}\right)^{4}} d t\right]^{1 / 2}}
\end{aligned}
$$


where $K^{\prime \prime}$ is a constant of integration. Consequently for this case we have the solution

$$
\begin{aligned}
& C_{1}=\frac{1}{b^{2}}\left(\frac{e^{2 t / b}\left(\dot{C}_{2} b^{2}+\dot{C}_{3}\right)}{\left(C_{2} b^{2}+C_{3}\right)^{3 / 2}\left[K^{\prime \prime}+\frac{4}{b^{2}} \int \frac{e^{(4 t / b)} C_{3}\left(2 C_{2} b^{2}-C_{3}\right)\left(\dot{C}_{2} b^{2}+\dot{C}_{3}\right)}{\left(C_{2} b^{2}+C_{3}\right)^{4}} d t\right]^{1 / 2}}-1\right) \\
& C_{2}=\frac{\dot{C}_{3} b \pm \sqrt{\dot{C}_{3}^{2} b^{2}-4 C_{3}\left(C_{3}+\dot{C}_{3} b\right)}}{2 b^{2}} \\
& C_{3}=\text { arbitrary function of time }
\end{aligned}
$$

Again an infinite class of solutions is possible.

\subsection{Case 4:}

This is the most general case and corresponds to the situation for which all of the coefficients $\mathcal{A}, \mathcal{B}, \mathcal{C}$ and $\mathcal{D}$ are nonzero. Equation (14) can be written as

$$
\dot{U}=-\frac{\mathcal{B}}{\mathcal{A}} U-\frac{\mathcal{C}}{\mathcal{A}} U^{2}-\frac{\mathcal{D}}{\mathcal{A}} U^{3}
$$

so that a variables separable equation is possible if $\frac{\mathcal{B}}{\mathcal{A}}, \frac{\mathcal{C}}{\mathcal{A}}$ and $\frac{\mathcal{D}}{\mathcal{A}}$ are constants. Then the solution may be written as the quadrature

$$
t-t_{0}=\int \frac{d U}{\frac{\mathcal{B}}{\mathcal{A}} U+\frac{\mathcal{C}}{\mathcal{A}} U^{2}+\frac{\mathcal{D}}{\mathcal{A}} U^{3}}
$$

It is important to emphasize that the additional constraints generated by $\frac{\mathcal{B}}{\mathcal{A}}, \frac{\mathcal{C}}{\mathcal{A}}$ and $\frac{\mathcal{D}}{\mathcal{A}}$ being constant simultaneously are not easy to simplify and in fact may not be consistent. Note that when $\mathcal{B}=0$ and $C_{2} b^{2}+C_{3}=\alpha$ we regain Case 1 with $\mathcal{A}=0$ and (13) becomes a Bernoulli equation. Then solution (17) is applicable. However, in general, when $\mathcal{B}=0$, the quadrature (23) is applicable.

\section{Discussion}

Herrera et al. [11] obtained the equation (9) governing the gravitational behaviour of a radiating spherical star undergoing shear-free gravitational collapse by imposing conformal flatness to the model. Investigations of this model have thus far been confined to exact solutions of linearised forms of this equation. The nonlinear behaviour of relativistic stellar models is an inherent part 
of realistic stars undergoing radiative gravitational collapse. A study of the physical features of these models hinges on the solution of the governing nonlinear equations. In this paper we have presented exact solutions of the governing equation in which the nonlinearity has been preserved. This has been effected by transforming the equation (9) into an Abel equation (14). We have found several classes of exact solutions given in (17), (18), (19) and (22) retaining the nonlinearity of the model. Note that these generate an infinite family of solutions which allow for a systematic study of radiating relativistic spheres in different scenarios.

It is important to observe that simple particular cases can be generated from our nonlinear models in Section 4. For example with $\mathcal{A}=0$ and $U \neq 0$ we may obtain the line element

$$
d s^{2}=B^{2}\left[-d t^{2}+d r^{2}+r^{2}\left(d \theta^{2}+\sin ^{2} \theta d \phi^{2}\right)\right]
$$

where (24) is in conformally flat form. Here for the simple case $C_{3}=\alpha$ in (17) we obtain $C_{1}=0, C_{2}=0$ and then $B^{2}$ is a constant; the Minkowski spacetime is regained. It is interesting to observe that the case $C_{1}=0$ in (17) also arises when $C_{3}$ takes the value

$$
C_{3}=\frac{2 \alpha^{2} \beta e^{-2 t / b}-\alpha}{2 \alpha \beta e^{-2 t / b}-3}
$$

where $\beta$ is a constant of integration and $C_{2}=\left(\alpha-C_{3}\right) / b^{2}$. Then we can write

$$
B^{2}=\left[\frac{b^{2}\left(2 \alpha \beta e^{-2 t / b}-3\right)}{\alpha b^{2}-2 \alpha r^{2}-2 b^{2} \alpha^{2} \beta e^{-2 t / b}}\right]^{2}
$$

This simple analytic form facilitates the analysis of the physical features of the model. We consider now some physical features which may be investigated in future work. With suitable choices of the arbitrary time functions, the luminosity radius

$$
L=(r B)_{\Sigma}
$$

may be easily found. The quantity

$$
\Gamma=\frac{d \ln p}{d \ln \rho}
$$

gives a measure of the dynamical instability of the stellar configuration at any given instant in time. We can use this result to confirm that the centre of the star is more unstable than the outer regions. Of particular importance is the thermal evolution of the fluid. The causal transport equation in the absence of rotation and viscous stress is

$$
\tau h_{a}^{b} \dot{q}_{b}+q_{a}=-\kappa\left(h_{a}^{b} \nabla_{b} T+T \dot{u}_{a}\right)
$$

where $h_{a b}=g_{a b}+u_{a} u_{b}$ projects into the comoving rest space, $T$ is the local equilibrium temperature, $\kappa(\geq 0)$ is the thermal conductivity, and $\tau(\geq 0)$ is the relaxation time-scale which gives 
rise to the causal and stable behaviour of the theory. As shown in Maharaj and Govender [12] for a physically reasonable radiative stellar model (25) becomes

$$
\beta(q B) T^{-\sigma}+A(q B)=-\alpha \frac{T^{3-\sigma}(A T)^{\prime}}{B}
$$

where $A$ and $B$ are the metric functions. Both the causal and noncausal solutions of (26) may be investigated in a simple model.

\section{Acknowledgements}

SSM thanks the National Research Foundation and the Durban University of Technology for financial support. SDM acknowledges that this work is based upon research supported by the South African Research Chair Initiative of the Department of Science and Technology and National Research Foundation.

\section{References}

[1] Santos NO. Non-adiabatic radiating collapse. Monthly Notices of the Royal Astronomical Society 1985; 216: 403-410.

[2] De Oliviera AKG, Santos NO, Kolassis CA. Collapse of a radiating star. Monthly Notices of the Royal Astronomical Society 1985; 216: 1001-1011.

[3] Govender M, Govinder KS, Maharaj SD, Sharma R, Mukherjee S, Dey TK. Radiating spherical collapse with heat flow. International Journal of Modern Physics D 2003; 12: 667-676.

[4] Kolassis CA, Santos NO, Tsoubelis D. Friedmann-like collapsing model of a radiating sphere with heat flow. Astrophysical Journal 1988; 327: 755-759.

[5] Di Prisco A, Herrera L, Esculpi M. Radiating gravitation collapse before relaxation. Classical and Quantum Gravity 1996; 13: 1053-1068.

[6] Di Prisco A, Falcon N, Herrera L, Esculpi M, Santos NO. Pre-relaxation processes in a radiating relativistic sphere. General Relativity and Gravitation 1997; 29: 1391-1405

[7] Herrera L, Martinez J. Gravitational collapse: a case for thermal relaxation. General Relativity and Gravitation 1998; 30: 445-471. 
[8] Govender M, Maharaj SD, Maartens R. A causal model of radiating stellar collapse. Classical and Quantum Gravity 1998; 15: 323-330.

[9] Govender M, Maartens R, Maharaj SD. Relaxational effects in radiating stellar collapse. Monthly Notices of the Royal Astronomical Society 1999; 310: 557-564.

[10] Govender M, Govinder KS. Causal solutions for radiating stellar collapse. Physics Letters A 2001; 283: 71-79.

[11] Herrera L, Le Denmat G, Santos NO, Wang G. Shear-free radiating collapse and conformal flatness. International Journal of Modern Physics D 2004; 13: 583-592.

[12] Maharaj SD, Govender M. Radiating collapse with vanishing Weyl stresses. International Journal of Modern Physics D 2005; 14: 667-676.

[13] Herrera L, Di Prisco A, Ospino J. Some analytical models of radiating collapsing spheres. Physical Review D 2006; 74: 044001. 